\title{
Preface
}

\section{Obstetrics and Gynecology Emergencies}

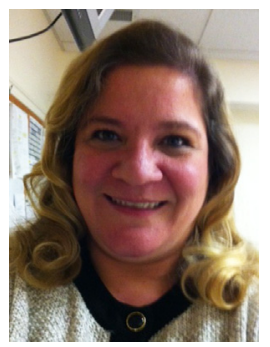

Susan E. Dantoni, MD, FACOG

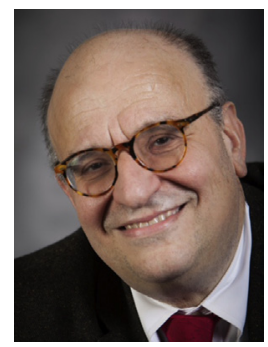

Peter J. Papadakos, MD, FCCM, FAARC Editors

This issue of Critical Care Clinics featuring Obstetrics and Gynecologic Emergencies began 20 years ago when a rather new obstetrician found herself knee deep in trouble with a patient experiencing an amniotic fluid embolism. Fortunately, the critical care team led by a very experienced intensivist arrived quickly, and because a multidisciplinary approach was taken, the patient experienced a very good outcome. This marriage of critical care and obstetrics led to many more collaborative efforts caring for very complicated patients. It also led to two children, three cats, a dog, a fish, and a horse, but that is another story!

While the vast majority of obstetric patients are delivered without incident, pregnancy remains a potentially high-risk condition with its own unique disease processes sometimes requiring the expertise of a multitude of clinicians. It is just as vital for the obstetrician to be educated regarding these medical complications as it is for other specialists, especially intensivists, to be aware of the pathophysiologic processes of pregnancy that may complicate the medical management of very sick gravida.

We have attempted to provide a broad review of critical care problems seen in obstetrics from anesthetic management to ethical issues. We have engaged experts to discuss neurologic, cardiac, renal, and hepatic complications of pregnancy. The topic of sepsis specifically addresses the complex and sometimes difficult conditions unique to pregnancy, which account for a significant amount of maternal morbidity and mortality throughout the world. The complex trauma issues join the trauma surgeon and the obstetrician in a battle to save both the mother and the fetus. This interplay of support of both parties is at the core of this aspect of critical care.

We are hopeful that this issue of Critical Care Clinics in which obstetric issues are covered is of interest not only to the critical care physician but also to the obstetric physician. 
This work is dedicated to our two children, Yanni and Ava, who have dealt with beepers, phone calls, and missed events. We love you both and appreciate your patience and understanding of our crazy careers.

Susan E. Dantoni, MD, FACOG Bellevue Women's Center/Ellis Hospital

Schenectady, NY, USA

Albany Medical College Albany, NY, USA

Peter J. Papadakos, MD, FCCM, FAARC

University of Rochester Rochester, NY, USA

E-mail addresses:

obdocsue@aol.com (S.E. Dantoni) peter_papadakos@urmc.rochester.edu (P.J.Papadakos) 